\title{
THREE-DIMENSIONAL STREAMLINED FINITE ELEMENTS: DESIGN OF EXTRUSION DIES
}

\author{
KEVIN R. J. ELLWOOD, T. C. PAPANASTASIOU AND J. O. WILKES \\ Department of Chemical Engineering, Dow Building, The University of Michigan, Ann Arbor, MI 48109, U.S.A.
}

\begin{abstract}
SUMMARY
A method to determine three-dimensional die shapes from extrudate swell and vice versa is presented using a three-dimensional Galerkin finite element method based on a streamlined formulation with the fluid velocities and pressures represented by triquadratic and trilinear basis functions respectively. The threedimensional streamlined method, an extension of the two-dimensional formulation, uses successive streamsurfaces to form a boundary-conforming co-ordinate system. This produces a fixed computational domain leaving the spatial location of the elements as unknowns to be determined with the standard primary variables $(u, v, w, p)$. The extrudate produced by a die of a given shape is considered for moderate Reynolds numbers. Finally, the method is extended to address the problem of die design, where a die profile is sought to produce a target extrudate shape.
\end{abstract}

KEY wORDS Streamlined finite elements Extrusion Free surface flows Die design Three-dimensional flows Three-dimensional finite elements

\section{INTRODUCTION}

Extrusion of non-Newtonian liquids through three-dimensional dies is a common practice in the polymer-processing industry. The extrusion process is used as an economical means of producing long strands or filaments of solid and annular cross-sections. ${ }^{1}$ Designing such extrusion dies requires an understanding of the liquid swelling or contracting as it leaves the die and deforms to its final shape, which is referred to as extrudate. The complicated fluid behaviour can be related to the rheology and processing conditions of the molten material as well as to the geometry of the die.

To obtain a desired final extrudate shape, the corresponding die shape must be determined. Present design procedures rely on empirical rules-of-thumb. An analytical description of the process would relieve some of the trial and error involved in designing and simulating extrusion. The first attempts to model the extrusion process focused on two-dimensional representations of planar and axisymmetric extrusions. ${ }^{2-5}$ These works solved the extrudate swell problem using a Picard iteration scheme to determine the location of the free surface. The iterative process, which exhibits linear convergence at best, ${ }^{6}$ used either the kinematic, the normal stress or the shear stress boundary condition to update the location of the free surface. Full Newton iteration would enable the location of the free surface to be determined along with the primary unknowns and also provide quadratic convergence. This procedure was first introduced for planar geometries ${ }^{7,8}$ and later for axisymmetric geometries as well. ${ }^{9}$

To date, three-dimensional studies have not received the same attention as two-dimensional cases. The earliest work that considered the three-dimensional extrusion appears to be that of 
Bush and Phan-Thien, ${ }^{10}$ in which the boundary integral element method was applied to study creeping flows of Newtonian liquids. Tran-Cong and Phan-Thien ${ }^{11}$ have extended this method to flows of viscoelastic liquids as well. Boundary methods reduce the dimensionality of the problem but cause iteration matrices to be full. The application of boundary integral methods can offer some advantages when the governing equations are linear; however, this advantage is lost when non-linearities such as non-Newtonian behaviour or finite convective terms enter the problem. The non-linear terms are usually treated as a distributed body force, which requires discretization and integration in the interior of the domain. The existence of integration in the interior means that a three-dimensional problem cannot be solved as a two-dimensional problem on the domain boundary, which is the case when the governing equations are linear. Additionally, treating the non-linear terms as a distributed body force makes it extremely difficult to implement a Newton iteration scheme. Recently, Karagiannis et al. ${ }^{12}$ used the finite element method with full Newton iteration, by extending the two-dimensional free surface parameterization technique of Kistler and Scriven ${ }^{7}$ to three dimensions, to study the extrusion of creeping Newtonian liquids. The problem of die design appears to have been considered by only Tran-Cong and Phan-Thien, ${ }^{13}$ who used the boundary element method and a Picard iteration scheme to update the die shape.

Here a method to solve three-dimensional free surface problems is presented using a Galerkin/ finite element method and the three-dimensional streamlines of the fluid to form the finite element tessellation. The actual location of the free surface and interior elements, and the fluid velocities and pressure are determined simultaneously. The advantage gained by determining all quantities simultaneously is that a Newton iteration scheme can be applied to both the standard extrusion and the reverse die design calculations, which exhibits the best convergence properties.

\section{GOVERNING EQUATIONS AND BOUNDARY CONDITIONS}

The flow configuration and co-ordinates for the extrusion of a non-Newtonian liquid are shown in Figure 1. The scales used for the length, velocity and stress are $L, U$ and $\mu U / L$, where $L$ is the width of the die, $U$ is the average fluid velocity at the inlet and $\mu$ is the fluid viscosity. The governing equations for this steady incompressible flow are the mass conservation equation

$$
\boldsymbol{\nabla} \cdot \mathbf{u}=\mathbf{0}
$$

and the momentum conservation equation

$$
\operatorname{Re} \mathbf{u} \cdot \nabla \mathbf{u}=\nabla \cdot \mathbf{T}+S t \mathbf{g} .
$$

$\mathrm{T}$ is the total stress tensor, which is related to the fluid deformation tensor $\mathbf{D}=(\nabla \mathbf{u})+(\nabla \mathbf{u})^{\mathrm{T}}$ by

$$
\mathbf{T}=-\mathbf{p I}+\mu\left[(\nabla \mathbf{u})+(\nabla \mathbf{u})^{\mathbf{T}}\right]=-\mathbf{p I}+\mu \mathbf{D} \text {. }
$$

The inflow and outflow boundaries are taken at finite distances $L_{1}$ and $L_{2}$ sufficiently far from the exit so that the flow is uniform at the outflow plane and fully developed at the inlet. To accommodate different die sections having a constant cross-section, the inflow boundary condition is given by the Poisson equation governing fully developed flow,

$$
\partial \tau_{x y} / \partial y+\partial \tau_{x z} / \partial z-\Delta P / \Delta L=0
$$

where $\Delta P / \Delta L$ is a constant pressure gradient, which is determined by the fact that the average dimensionless $u$-velocity component at the inlet is unity or

$$
\int_{\text {inlet }} u \mathrm{~d} S / \int_{\text {inlet }} \mathrm{d} S=1
$$




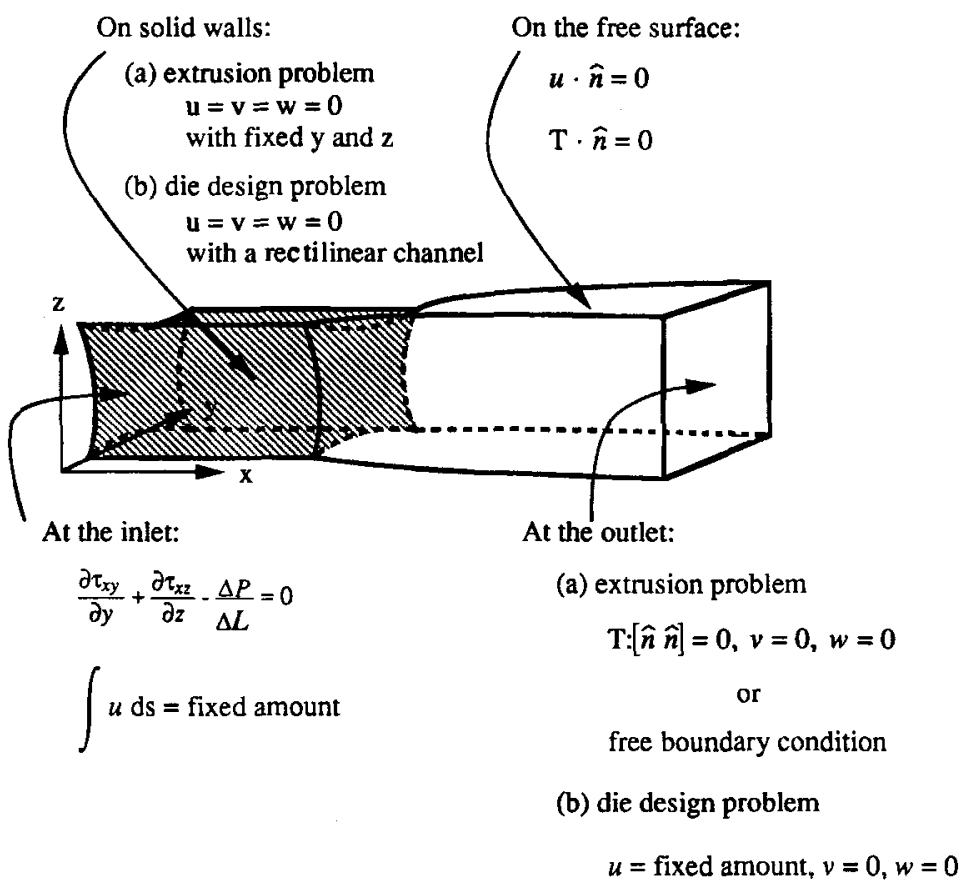

Figure 1. Three-dimensional extrusion: domain, co-ordinates and boundary conditions

For the case where liquid is extruded from a fixed die (the extrusion formulation), the $x$ component of velocity, $u$, can be determined a priori by solving equations (3) and (4). However, when the final extrudate dimensions and flow rate are fixed and the die geometry is sought (the die design formulation), the inlet velocity profile and the linear pressure drop must be determined along with all the other primary unknowns, since their magnitude changes with the die crosssection during Newton iteration.

There are several choices for the condition to be applied at the synthetic outflow plane. At an infinite distance downstream the boundary condition for the outflow in the absence of gravity and surface tension is

$$
-p+\tau_{\hat{\mathrm{An \hat {ी }}}}=0, \quad v=0, \quad w=0,
$$

where $\hat{\mathbf{n}}$ is the outward normal to the outflow plane and $\tau_{\hat{\mathrm{n}} \hat{\mathrm{n}}}$ is the normal component of the viscous stress tensor. However, if gravity or surface tension is present or if the synthetic outflow plane is placed at a point where the flow is not uniform, there is no such natural boundary condition. This boundary condition is safely formulated using the concept developed by Malamataris, ${ }^{17}$ in which the validity of the weak form of the governing equations (equation (10b)) is extended to the 'synthetic' outflow boundary, instead of replacing them there with essential or natural boundary conditions, which are not accurate at the specific boundary. This 'free' boundary condition minimizes the energy functional among several possible outflow boundary conditions for creeping flows and appears to be the most appropriate boundary condition at the synthetic boundary. Malamataris ${ }^{17}$ used the free boundary condition for various problems that encounter truncated domains and found that the solutions completely agree with the results obtained by considering the complete domains, even when the synthetic 
boundary cuts through regions of recirculation and eddies. Furthermore, they showed that this free boundary condition is a relation between the variation of the weighted quantity and its flux close to the synthetic outflow. The same principles were extended successfully to unsteady film flows on unbounded domains recently by Malamataris. ${ }^{17}$ The free boundary condition has been applied to the case where extrusion is under the influence of gravity, which is discussed in detail in the results section. All other cases were computed by applying equation (5) as a boundary condition.

A free surface is formed as the liquid leaves the die. A momentum balance at the free surface shows that the total normal stress is balanced by capillary forces. If the effects of surface tension are neglected, the boundary condition on the free surface is

$$
\mathbf{T} \cdot \hat{\mathbf{n}}=0 \text {. }
$$

The location of the free surface must be determined as part of the unknowns in the problem and this can be done by using any of several different criteria. The most straightforward method is to use the fact that there is no mass penetration across the free surface or the normal component of velocity is zero; thus

$$
\mathbf{u} \cdot \hat{\mathbf{n}}=\mathbf{0}
$$

applies along the free surface, as shown in Figure 2. Alternatively, one can use the fact that a differential increment in the position vector $r$ which defines the streamlines bounding the streamsurface is parallel to the velocity vector $\mathbf{u}$ along the streamline:

$$
\mathrm{dr} \times \mathbf{u}=\mathbf{0} \text {. }
$$

Equation (8) leads to the following scalar equations, which can be used to determine the location of streamlines:

$$
\begin{gathered}
\mathrm{d} y / v=\mathrm{d} x / u, \\
\mathrm{~d} z / w=\mathrm{d} x / u
\end{gathered}
$$

\section{FINITE ELEMENT FORMULATION}

The flow domain was tessellated using rectangular Lagrangian elements as shown in Figure 3. The fluid velocity and pressures are represented by triquadratic, $\phi^{i}(\xi, \eta, \zeta)$, and trilinear, $\psi^{i}(\xi, \eta, \zeta)$, basis functions,

$$
\mathbf{u}=\sum_{1}^{n} \phi^{i} \mathbf{u}_{i}, \quad p=\sum_{1}^{n} \psi^{i} p_{i},
$$

respectively, with $\xi, \eta$ and $\zeta$ being local isoparametric co-ordinates having the range $[-1,1]$. The unknowns $u_{i}$ and $p_{i}$ are determined by demanding that the residuals equations are orthogonal to the basis functions $\phi^{i}$ and $\psi^{i}$, which is referred to as the Galerkin/finite element method. The final algebraic system to be solved is represented by

$$
\begin{gathered}
-R_{p}^{k}=\int_{v} \psi^{k} \nabla \cdot \mathbf{u} \mathrm{d} v=0, \\
-R_{\mathbf{v}}^{k}=\int_{v} \phi^{k}(\operatorname{Reu} \cdot \nabla \mathbf{u}-S t \mathbf{g}) \mathrm{d} v+\int_{v} \nabla \phi^{k} \cdot \mathbf{T} \mathrm{d} v-\underbrace{\int_{s}^{\phi^{k} \mathbf{T} \cdot \hat{\mathbf{n}} \mathrm{d} s}=0 .}_{\text {surface integrals }}
\end{gathered}
$$




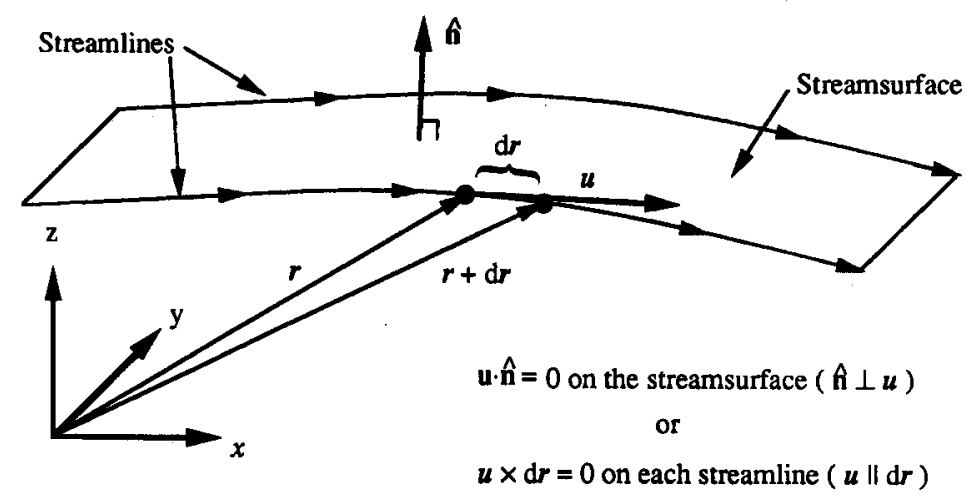

Figure 2. Defining equations for streamlines/streamsurface. A streamsurface is defined by using the fact that the component of velocity perpendicular to the streamsurface is zero. Alternatively, three-dimensional streamlines can be defined by using the fact that a differential change in its position vector, dr, is parallel to the velocity vector $u$

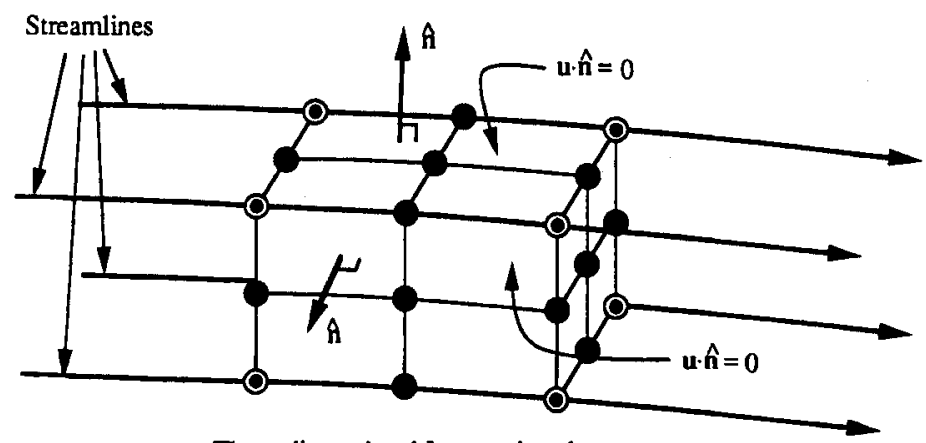

Three-dimensional Lagrangian element.

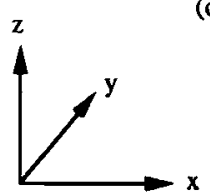

(only visible nodes are shown)

$u, v, w, x$, and $y$ are unknown

( $u, v, w, x, y$, and $p$ are unknown

Figure 3. A typical 3D streamlined element, defined by four movable streamlines (moving $y$ and $z$ ) and two fixed planes (fixed $x$ )

The divergence theorem has been applied in formulating equation (10b) to incorporate natural boundary conditions.

The free boundary condition is implemented by simply evaluating the stress tensor $\mathbf{T}$ in the surface integrals of the momentum equations $(10 \mathrm{~b})$ in terms of the, as of yet, unknown outflow nodal values of $u, v, w, y, z$ and $p$ along with the volume integrals. This is equivalent to extending the weighted momentum to the synthetic outflow plane.

In the application of finite element algorithms some type of mesh construction must take place. The importance of mesh construction is even more relevant when flows are three-dimensional with free surfaces, because mesh construction can be quite time-consuming. There are several different schemes that can be used to map the computational domain into a fixed region leaving 
the location of any free surfaces as unknowns. Some of the more popular schemes include elliptic mesh generation ${ }^{14}$ and parametric splines. ${ }^{7,12}$ Streamlined elements have been used in the past to solve steady two-dimensional flows of fluids with memory. ${ }^{15,16}$ In this study the concept of streamlined elements has been generalized to flows in three dimensions.

In two-dimensional flows streamlines are lines along which flow rates are constant; for steady flows they correspond to the paths traced of imaginary massless particles. In three-dimensional flows the concept of streamlines can be extended to streamsurfaces, which are surfaces separating volumes of constant flow rates and are illustrated in Figure 3. The intersections of different streamsurfaces are streamlines, which can be used to form a three-dimensional mesh.

In this procedure the two-dimensional sides of elements coincide with streamsurfaces that are not explicitly known a priori. The sides of the elements can be determined simultaneously with the velocity field by knowing that the sides of the elements are actually streamsurfaces. As discussed earlier, there are two approaches to determining the locations of streamlines. The first of these approaches is to use the fact that there is no mass penetration along the surface (or streamsurface). Therefore the Galerkin algebraic equation governing the spatial location of the sides of each element is

$$
R_{\mathbf{x}}^{k}=\int_{s} \mathbf{u} \cdot \hat{\mathbf{n}} \phi^{k} \mathrm{~d} s=0
$$

where $\mathbf{x}=\sum_{1}^{n} \phi^{i} \mathbf{x}_{i}$ and $\phi(\xi, \eta, \zeta)$ is evaluated at $\eta=(0,1)$ and $\zeta=(0,1)$. Equation (11) is a relationship between the position of the element, $\hat{\mathbf{n}}(\mathbf{x})$, and the velocity $\mathbf{u}$; thus $\mathbf{x}$ and $\mathbf{u}$ must be determined simultaneously. Note that the quantity $x_{i}$ has the components $\left(x_{i}, y_{i}, z_{i}\right)$ and equation (11) is used to determine only the $y_{i}$ - and $z_{i}$-components of $x_{i}$. The $x_{i}$-component of $x_{i}$ is fixed a priori, although it could be movable too, if needed, to satisfy an imposed constraint. The second approach to define the unknown element nodal locations is to use the fact that they are on streamlines, which allows equations (9) to be integrated directly:

$$
\begin{aligned}
& y-y_{0}=\int \frac{v}{u} \mathrm{~d} x, \\
& z-z_{0}=\int \frac{w}{u} \mathrm{~d} x .
\end{aligned}
$$

Both schemes were used in the streamlined code and equation (11) was found to give better convergence properties. However, equations (12) allow a direct method of determining the locations of streamlines when an initial guess is made for the velocity profile.

All the Galerkin residuals are evaluated numerically using nine-point Gaussian integration. The resulting set of non-linear algebraic equations is solved by Newton-Raphson iteration, which has the form

$$
\mathbf{J} \cdot \Delta \mathbf{q}^{n}=-\mathbf{R}\left(\mathbf{q}^{n}\right)
$$

where $\mathbf{q}=\left[u_{1}, v_{1}, w_{1}, y_{1}, z_{1}, p_{1}, \ldots, u_{N}, v_{N}, w_{N}, y_{N}, z_{N}, p_{N}\right]$ is the vector of unknowns and $\mathbf{J} \equiv \partial \mathbf{R} / \partial \mathbf{q}$. Equation (13) is a system of linear equations which was solved repeatedly until the maximum relative update $\Delta q^{n}$ was less than $10^{-4}$. To solve the linear system represented by equation (13), the frontal method developed by Hood ${ }^{18}$ was optimized in two ways.

1. To accommodate the capabilities of modern vectorized and parallel processing computers, the vectorization techniques described by Wolfe $^{19}$ were applied to major loop structures. 
2. To eliminate wasteful multiplications by zero, an indirect addressing scheme, which referred to non-zero entries in a given row, was used. Computing the index for non-zero entries caused very little overhead in the assembly and elimination procedure and greatly reduced the time required for back substitution.

Additionally, it was found that loops in the assembly routine could be effectively vectorized, reducing the time required to form the element stiffness matrix.

\section{RESULTS AND DISCUSSION}

Initially, extrusion of a creeping Newtonian liquid from an axisymmetric die was studied in order to test the finite element scheme. Figure 4 shows the extrusion process modelled as a threedimensional jet with symmetry being assumed at the $y=0$ and $z=0$ planes. The initial guess for the velocity profile was obtained by assuming the free surface was a continuation of the die profile, which was fixed for the first iteration. The die swell is clearly evident in Figure 4 and the final swell ratio of $1 \cdot 14$ compares well with data available in the literature. ${ }^{9}$

Three-dimensional jet data are rare in the literature for comparison purposes. However, some data exist for the extrusion of liquids out of a square die. ${ }^{1}$ Figure 5 shows the extrusion of a Newtonian liquid from a square die for vanishingly small Reynolds numbers $R e$ and neglecting surface tension. The maximum swelling occurs along the flat sides where the fluid swells by about $19 \%$, which agrees with the computations of Karagiannis et al. ${ }^{12}$ and the experiments of Michaeli. ${ }^{1}$ The swelling of the fluid as it leaves the die occurs due to the sharp transition of the velocity as it leaves the die, which is shown in Figure 6. Inside the die the fluid is subject to a no-slip boundary condition at the walls, which changes to zero normal stress at the free surface formed as the fluid leaves the die. The change in boundary conditions causes the fluid to make a

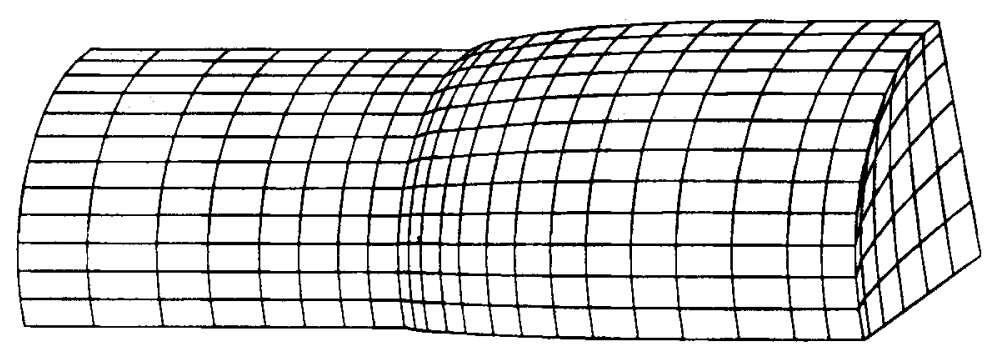

Figure 4. The extrusion of a creeping Newtonian liquid from an axisymmetric die is used as a benchmark solution. The jet is shown to swell as it leaves the die, yielding a swell ratio of $1 \cdot 14$. Only one-quarter of the jet is shown

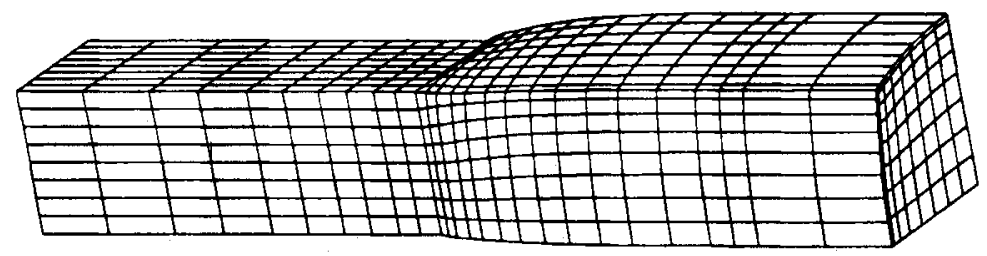

Figure 5. The extrusion of a Newtonian liquid from a square die is shown for $S t=0$ and $R e=0$. The results for $R e=0$ compare well with the simulations of Karagiannis et al. ${ }^{12}$ as well as the experimental results of Michaeli. ${ }^{1}$ Notice that only one-quarter of the jet is shown 

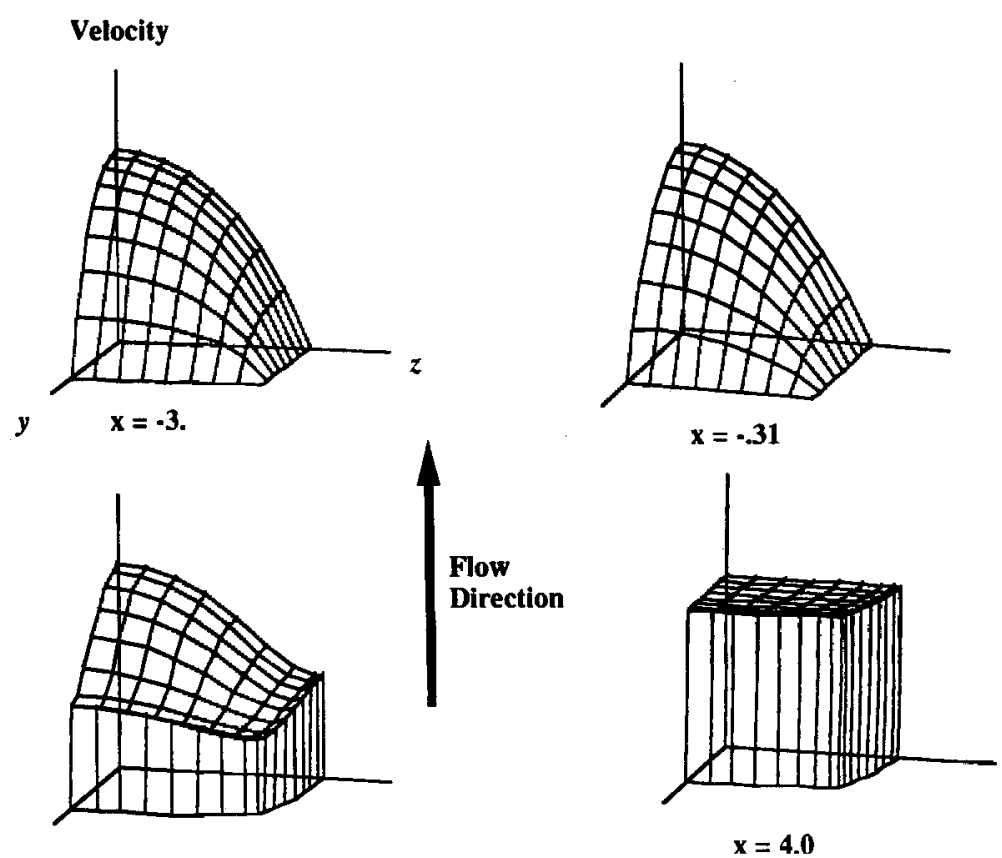

$x=0.366$

Figure 6. The transition of the $x$-component of the velocity as it leaves the die is shown at several position along the flow field. Note that the square die exit is located at $x=0$
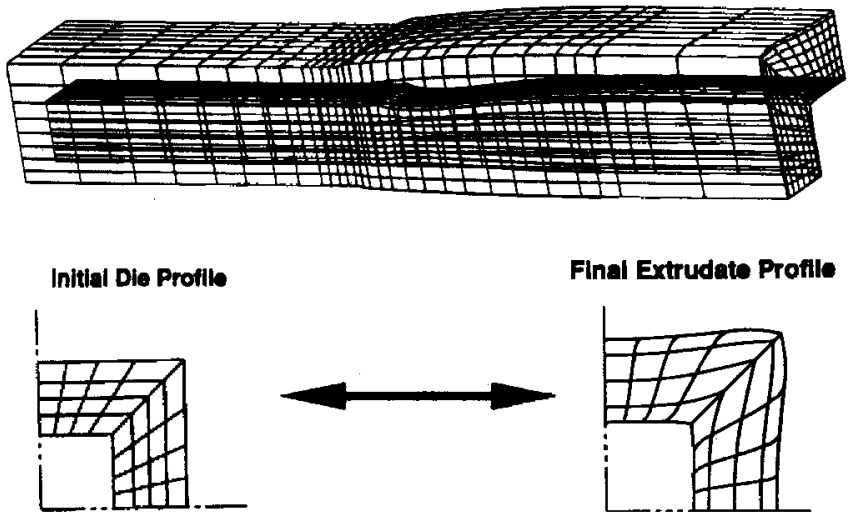

Figure 7. The extrusion of a Newtonian liquid from a square annular die is shown for $R e=0$ and $S t=0$. The maximum swelling is shown to be at the corners of the square annulus. Notice that only one-quarter of the jet is shown

transition from a 'parabolic' profile inside the die to a flat profile outside the die, which give rise to the normal forces that expand the jet.

The extrusion of liquids through annular dies is also of great industrial importance. Such an example is shown in Figure 7, where the maximum swelling occurs in the corner regions. Again the complex flow rearrangement as the fiuid makes the transition from fully developed flow in the annulus to the free annular jet is responsible for the corner swelling. Also shown in Figure 7 are 
the finite element tessellations at the die entrance and at the outflow boundary, which illustrate how the inlet mesh is modified by the streamlined technique in order to keep successive elements in the same streamtube bounded between the same streamlines and streamsurfaces.

The predicted extrusion profiles for a Newtonian liquid produced from a square die are shown in Figure 8. A finite Reynolds number $R e$ has the effect of contracting the extrudate, which has also been shown in the two-dimensional cases of axisymmetric and planar flows. ${ }^{9}$ The base case shown in Figure 8 is extrusion at $R e=1$. When the Reynolds number increases to $R e=5$, the swelling is reduced along the sides of the jet and the corner region begins to contract. As the Reynolds number is increased further to $R e=20$, the swell at the centre of the sides vanishes accompanied by a further increase of contraction in the corner region.

The plug flow boundary condition, equation (5), was used at the outflow of the previous simulations because it is known that the free jets will attain this configuration far from the nozzle exit. However, there are cases where the outflow boundary condition is unknown, such as transient jet flows or the flow of a jet under the influence of gravity. In such cases the free boundary condition described earlier was used. Figure 9 illustrates the extrusion of a fluid under the influence of gravity. The fluid accelerates as it leaves the die, causing the jet to become thinner. This result agrees qualitatively with the two-dimensional simulations performed by Georgiou et al. ${ }^{9}$ who assumed that the jet would reach a final uniform velocity profile far from the die exit.

The problem of die design appears to have been considered by only Tran-Cong and PhanThien using the boundary element method and a crude Picard iteration scheme to update the die

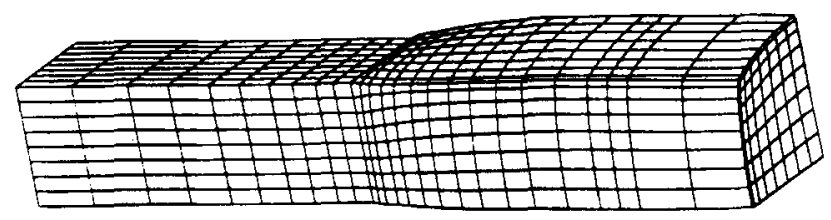

(a) Reynolds number $R e=1.0$

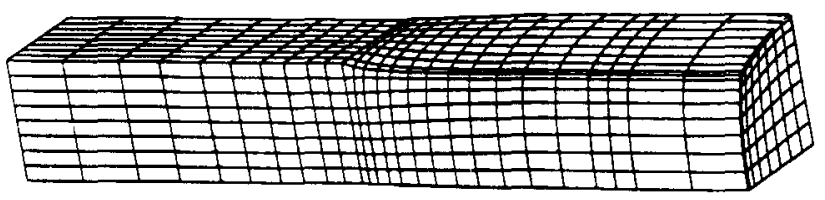

(b) Reynolds number $R e=5.0$

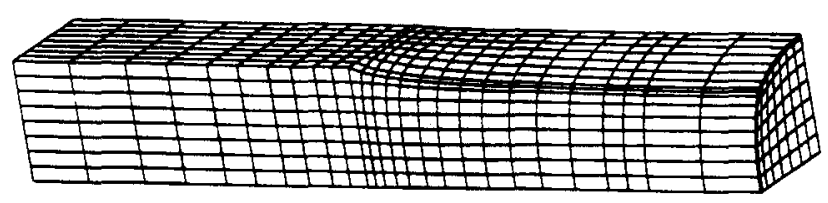

(c) Reynolds number $\operatorname{Re}=\mathbf{2 0 . 0}$

Figure 8. The extrusion of a Newtonian liquid from a square die is shown for $S t=0$ and (a) $R e=1$, (b) $R e=5$ and (c) $R e=20$ 


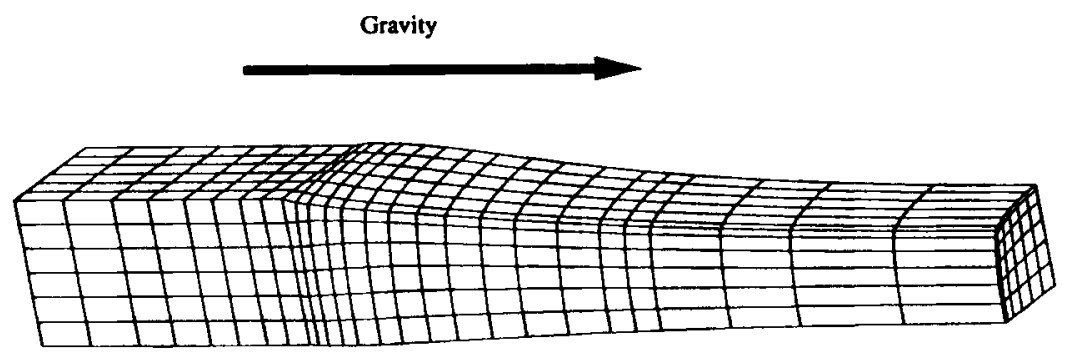

Figure 9. The extrusion of a Newtonian liquid from a square die is shown for $R e=0$ and $S t=0 \cdot 5$. The jet is seen to become thinner as the fluid accelerates away from the nozzle

shape. ${ }^{13}$ This type of simulation is of great practical importance to the polymer-processing industry because it is often required to produce plastic parts with a diversity of shapes by the extrusion process. Present practice in die construction consists of trial-and-error guesses at the correct die profile to produce a target extrudate. The die orifice is usually undersized initially and is enlarged slowly until the desired extrudate dimensions are reached. This start-up procedure can account for up to $50 \%$ of the total die cost. ${ }^{1}$ For complicated extrudate geometries no initial guess for the die profile can be made and for simple geometries a substantial cost reduction can be obtained with an initial die profile that is close to the actual profile needed to produce the proper extrudate.

Here we consider problems where the final jet dimension or target extrudate shape is a square or a 2:1 rectangle and we need to compute the die geometry that will produce it. The initial guess for the Newton-Raphson procedure was produced by the following scheme.

1. The standard extrusion problem was solved for a die with the shape of the final target extrudate shape to obtain a guess for the velocity profile for the extrudate with the desired extrudate profile.

2. The nodes located on the outflow plane of the extrusion problem were translated to new nodal locations on the outflow plane of the die design problem. These new nodal points are ends of streamlines along which the velocity is assumed to be the same velocity computed for that streamline before it was translated.

3. Knowing the end point of a streamline with an assumed velocity along the streamline, the initial guess for the die shape was obtained by backward integration along all streamlines using equations (12). The guess for the die shape will be the location $(y, z)$ of the free surface streamlines at the plane that divides the die section and the free surface flow, $x=0$.

The results are shown in Figures 10 and 11, where the final die profiles are shown. In both figures the sides of the dies are curved inwards to counterbalance the swelling effects that normally occur along flat sides. Also shown in Figures 10 and 11 is the extrudate shape that would be obtained if the die profile was assumed to be that of the desired 'target' extrudate shape. It is clearly shown that a square or rectangular die would produce an extrudate much different from the desired square or rectangular shape. One could intuitively guess that the sides of the die required to produce a square jet should be curved inwards by examining Figure 5 . However, such intuition would not be available for more complicated geometries. Using the streamline finite element method, we were able to directly compute the die shape a priori, which saves much trial-and-error guessing as well as start-up costs when performing the design. 


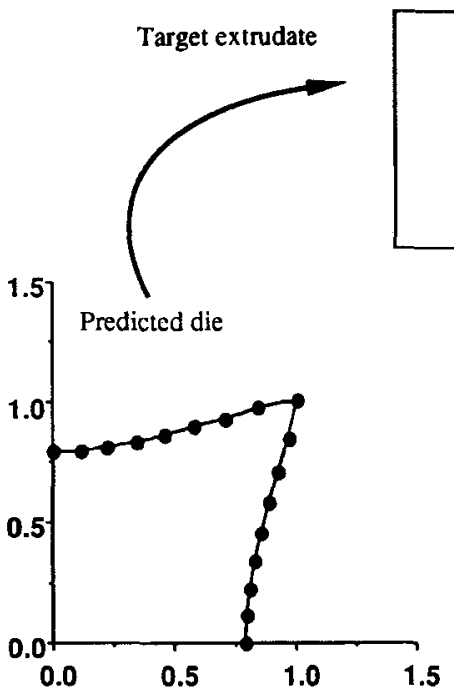

Die needed to produce a square

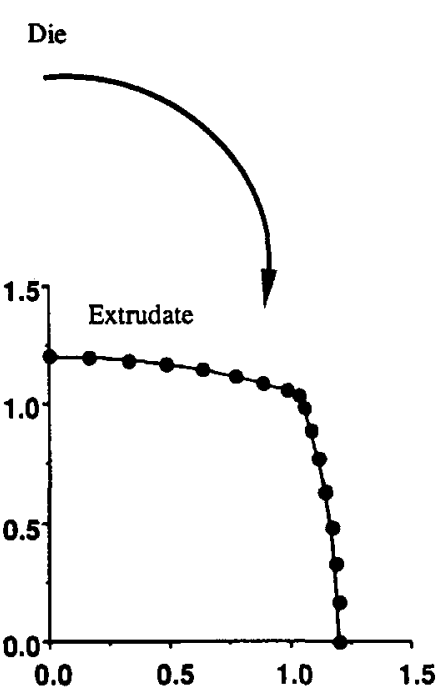

Extrudate profile from a square die

Figure 10. The die or nozzle shape is shown so that extrusion of a Newtonian liquid with $R e=0$ will form a square final target configuration. A square die would give an entirely different extrudate

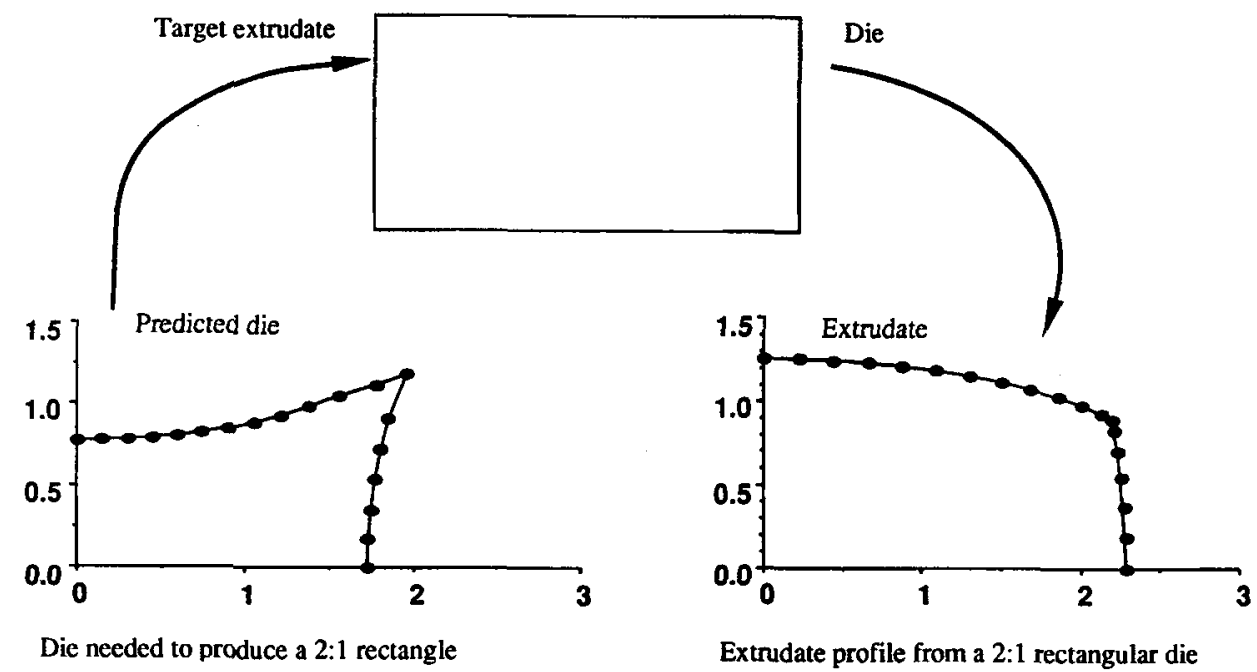

Figure 11. The die or nozzle shape is shown so that extrusion of a Newtonian liquid with $R e=0$ will form a $2: 1$ rectangle. A 2:1 rectangular die would give an entirely different extrudate

\section{CONCLUSIONS}

Extrusion of Newtonian liquids from three-dimensional dies has been studied using a streamline Galerkin/finite element method and full Newton iteration. The streamline formulation is particularly convenient to generate the tessellation, to keep track of three-dimensional material surfaces that enter in three-dimensional free surface flows and to facilitate book-keeping, which is rather involved with three-dimensional free surface flow calculations. 
Resulting three-dimensional extrudate shapes from rectangular and annular dies were computed. Inertial effects were shown to reduce swelling along 'flatter' sides and to increase contraction of corner regions. Extruding under the influence of gravity, modelled by using a free boundary condition at the outflow plane, results in contraction of three-dimensional extrudates.

Along with the direct extrusion computations, the inverse problem of designing the die, given the final target extrudate, was considered. Shapes of dies required to produce extrudates of square and rectangular sections were computed.

\section{ACKNOWLEDGEMENTS}

The authors wish to acknowledge that this research was sponsored by grants-in-aid from General Motors Corporation. All computational results were obtained on the IBM 3090-600E/VM and the Alliant FX/8 at the University of Michigan.

\section{REFERENCES}

1. W. Michaeli, Extrusion Dies: Design and Engineering Computations, Hanser, New York, 1984.

2. R. E. Nickell, R. I. Tanner and B. Caswell, 'The solution of viscous incompressible jet and free-surface flows using finite-element methods', J. Fluid Mech., 65, 189 (1974).

3. K. R. Reddy and R. I. Tanner, 'Finite-element solution of viscous jet flows with surface tension', Comput. Fluids, 6, 83 (1978).

4. B. J. Omodei, 'Computer solutions of a plane Newtonian jet with surface tension', Comput. Fluids, 7,79 (1979).

5. B. J. Omodei, 'On the die-swell of an axisymmetric Newtonian jet', Comput. Fluids, 8, 275 (1980).

6. W. J. Silliman and L. E. Scriven, 'Separation flow near a static contact line: slip at a wall and shape of a free surface', $J$. Comput. Phys., 34, 287 (1980).

7. S. F. Kistler and L. E. Scriven, 'Coating flow theory by finite-element and asymptotic analysis of the Navier-Stokes system', Int. j. numer. methods fluids, 4, 207 (1984).

8. H. S. Kheshgi and L. E. Scriven, 'Penalty finite-element analysis of unsteady free-surface flows', in R. H. Gallagher, J. T. Oden, O. C. Zienkewicz, T. Kawai and M. Kawahara (eds), Finite Elements in Fluids, Wiley, New York, 1984, Chap. 19.

9. G. C. Georgiou, T. C. Papanastasiou and J. O. Wilkes, 'Laminar Newtonian jets at high Reynolds numbers and surface tension', AIChE J., 34, 1559 (1988).

10. M. B. Bush and N. Phan-Thien, 'Three-dimensional viscous flows with a free surface: flow out of a long square die', J. Non-Newtonian Fluid Mech., 18, 211 (1985).

11. T. Tran-Cong and N. Phan-Thien, 'Three-dimensional extrusion process by boundary element method: II. Extrusion of a viscoelastic fluid', Rheol. Acta, 28, 1(989).

12. A. Karagiannis, A. N. Hrymak and J. Vlachopoulos, 'Three-dimensional extrudate swell of creeping Newtonian jets', AIChE J., 34, 2088 (1988).

13. T. Tran-Cong and N. Phan-Thien, 'Die design by a boundary element method', J. Non-Newtonian Fluid Mech., 30(1), $37-46$ (1988).

14. J. F. Thompson, Z. U. A. Warsi and C. Wayne Mastin, Numerical Grid Generation: Foundations and Applications, North-Holland, New York, 1985.

15. R. D. Wesson and T. C. Papanastasiou, 'Flow singularity and slip velocity in plane extrudate-swell computations', J. Non-Newtonian Fluid Mech., 26, 277 (1988).

16. A. C. Papanastasiou, C. W. Macosco and L. E. Scriven, 'Streamlined finite-elements and transit times', in R. H. Gallagher, G. F. Garey, J. T. Oden and O. C. Zienkiewicz (eds), Finite Elements in Fluids, Vol. 6, Wiley, New York, 1985 , pp. 263-278.

17. N. Malamataris (with T. C. Papanastasiou), 'Computer-aided analysis of flows on moving and unbounded domains: phase-change fronts and liquid leveling, Ph.D. Thesis, University of Michigan, 1991.

18. P. Hood, 'Frontal solution program for unsymmetric matrices', Int. j. numer. methods eng., 10, 379 (1976).

19. M. Wolfe, 'Vector optimization vs vectorization', J. Parallel Dist. Comput., 5, 551 (1988). 